\title{
The Uniaxial Constitutive Models of Reinforcement and Concrete for Nonlinear Dynamic Analysis
}

\author{
Hu Q1 ${ }^{1, a^{*}}$, Yun-Gui LI,b \\ ${ }^{1}$ Beijing Shunyi District Linhe Street No. 15, China \\ aqihu_810@163.com, 'liyungui@china.com \\ ${ }^{*}$ Corresponding author
}

Keywords: Concrete Constitutive Model, Elastic Plastic Constitutive Model, Elastic Plastic Damage Model.

\begin{abstract}
The constitutive model of materials is essential to nonlinear analysis, in this paper, uniaxial constitutive models of reinforcement and concrete are summarized, and some meaningful uniaxial models of concrete and reinforcement are proposed.
\end{abstract}

\section{Introduction}

Reinforced concrete structure is a composite material made of plain concrete and steel bars. Concrete materials are more complicated than relatively uniform metal material in Mechanical properties. Therefore, the cognition of the characteristics of concrete constitutive is directly related to the economical and rationality of the structure design.

Based on the present research situation on concrete constitutive, it is can be objectively evaluated that: although concrete material constitutive theories including experimental research and theoretical simulation, has made great progress, the theories still can not clearly describe some important constitutive characteristics of concrete.

Concrete constitutive theory research is of important theory significance and engineering value, but there is still some blank in the filed which needs our further study. In this article, the application and the development of the current concrete and rebar constitutive models have been stated, and a number of new concrete and rebar constitutive model have been established, providing reference for practical engineering.

\section{D Uniaxial Constitutive Model}

\section{Concrete Uniaxial Constitutive Model}

Uniaxial constitutive model of concrete is the most basic constitutive model, as well as the basis for multi-axis[1]. In the nonlinear analysis of reinforced concrete structures, the uniaxial constitutive models are essential factors, determining the accuracy of the results.

Scholars have done a lot of research on the concrete under uniaxial hysteresis loading, among which representative uniaxial hysteretic constitutive models of concrete are: Bolong Zhu model (1980)[2], Guo-Zhang model (1981)[3], Blakeley model (1973)[4], Mander model (1988)[5], Teng Zhiming-Zou Lixiang model (1996) [6], Saatcioglu model (1992) [7].

1)Zhu Bolong (1980) model: Zhu Bolong (1980) proposed a model, as shown in Fig.1, the model not only gives the concrete compressive unloading, loading curve, but also can consider the concrete tensile cracking after compression crack effect, it is a comprehensive model.

2)Guo - Zhang (1981) model: Guo - Zhang (1981) model is of accurate calculation and can reflect a lot of nonlinear characteristic of concrete like stiffness degradation, residue plastic deformation, as shown in Fig.2. The model's main weakness is that it's only suitable to concrete compressive segment but for the reloading after cracking. 
3)Blakeley model: In 1973, Blakeley and Park put forward a concrete uniaxial hysteretic constitutive model which use Kent-Park model as the skeleton curve, as shown in Fig.3. In this model, unloading, loading curves are simplified as straight lines.

4)Mander (1988) model: Mander (1988) proposed a model which is suitable for different stirrup forms, and uses an equation to describe skeleton curve, and gives the loading-unloading hysteresis rule, as shown in Fig.4.

5)Focal Point (1989) Model: Yankelevsky, Reinhardt put forward concrete compression hysteretic constitutive model in 1987 and tensile hysteresis model in 1989. The model reflects the basic character of the concrete performance in repeating hysteresis loading, and uses a series of focus and given points to identify the unloading and re-loading and curve with convenient calculation.

6)Teng -Zou (1996) model: Zhiming Teng, and Lixiang Zou (1996) simplified focal point model and added a part of hysteretic rules, as shown in Fig.5.

7)Saatcioglu (1992) model: Saatcioglu and Razvi (1992) proposed a model. The model uses different curve equations to describe the strain-stress relations of common and high strength concrete under reinforce confine. The model is a comprehensive model, which not only considers confinement effect to core concrete caused by the section form, transverse stirrup forms, transverse stirrup strength, horizontal reinforcement number and spacing, but also proposes a calculation method for utmost strain of confine and unconfined concrete.
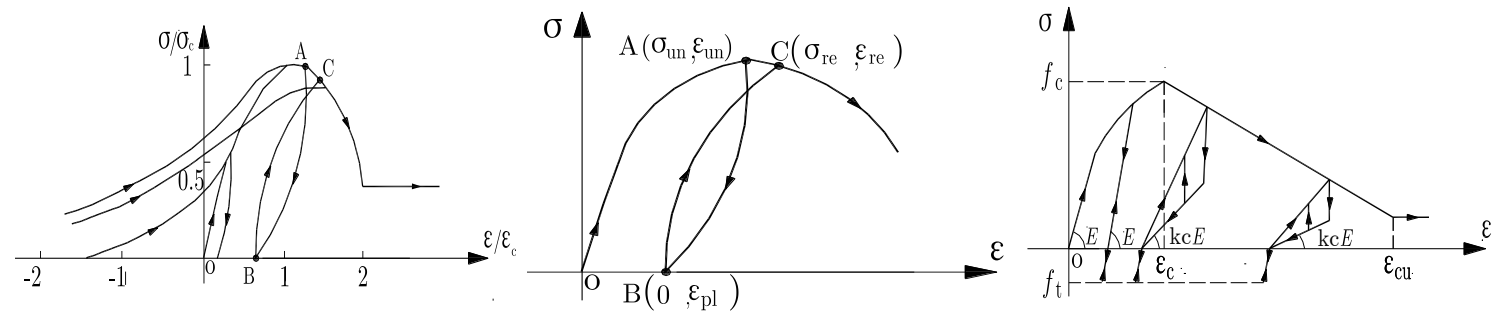

Fig. 1 Zhu Bolong (1980) Model Fig. 2 Guo - Zhang (1981) Model Fig. 3 B1akeley Model

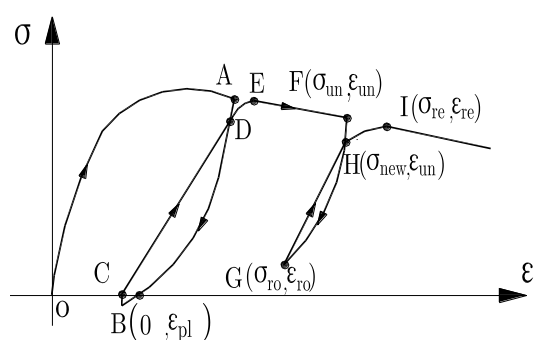

Fig. 4 Mander (1988) Model

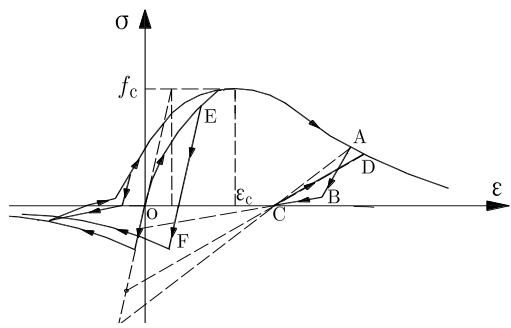

Fig. 5 Teng -Zou (1996) Model

The constitutive model for confined concrete under uniaxial hysteresis load had been studied in detail, among which six kinds of typical models had been analyzed by the use of ABAQUS UMAT. Through the comparison between the models calculation results and experimental results and the comparison between the various models, accuracy and computational efficiency of each model had been studied in three facts: compression skeleton curve, compression hysteresis curve, and tension hysteresis curve.

(1)For the rise section of skeleton curve, the initial elastic modulus of Zhenhai Guo, Xiuqin Zhang model remain unchanged as the stirrup ratio increased, and the calculation result fits the test well. Compared with Guo Zhenhai, Zhan Xiuqin model, Mander model is of high computational efficiency.

(2)For the descent section of skeleton curve, B1akeley model, Saatcioglu model, Mander model are smooth and more suitable for the analysis of circular section member, while Zhang model, Teng 
- Zou model are more suitable for the analysis of rectangular section. Saatcioglu model using straight line has the best computational efficiency but with slightly worse precision. Mander model and Guo-Zhang model are of similar calculation efficiency. Mander model has a uniform formulation to describe skeleton curve with best stability.

(3)For hysteretic curve under uniaxial compression loading, Mander model, Bolong Zhu model, Guo-Zhang model all can simulate stiffness degradation of concrete material, but Mander model has highest accuracy, Mander model, Guo-Zhang model are very close when unloading strain was little, but when unloading high strain, unloading stiffness of Guo-Zhang model is even greater than the initial elastic stiffness, in consistent with the practice.

(4)Both Bolong Zhu model and Teng -Zou model include tension hysteretic curve. However,

Teng -i

Fig.t

model,

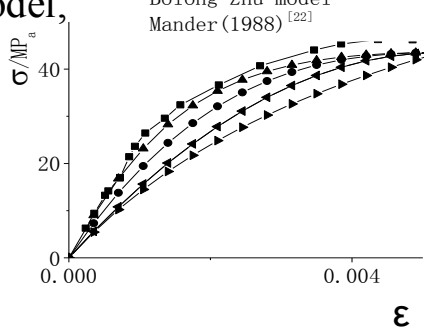
ensi $n$ of the of the $\mathrm{u}$

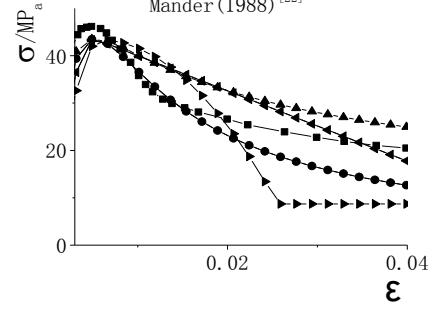
e path of stain-tress loading. nd the skeleton curra $\underset{\text { Guo-Zhang model }}{\text { f cal }}$ re models.

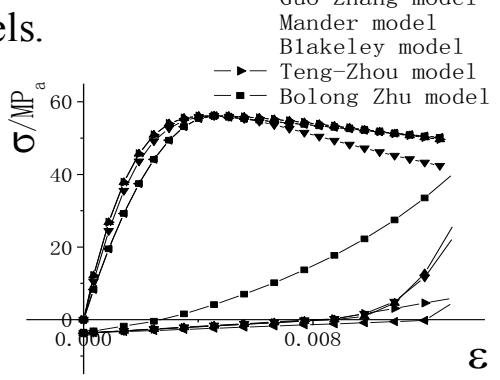

Fig.6 Comprasion of Skeleton Curve Fig.7 Comprasion of Skeleton Curve Fig.8 Comparison of Unload Curve

Hu Qi and Yungui Li[8] proposed a practical concrete uniaxial constitutive model for hysteretic loading with the ability of considering complex loading paths through comparative analysis of the above models(Fig.9).
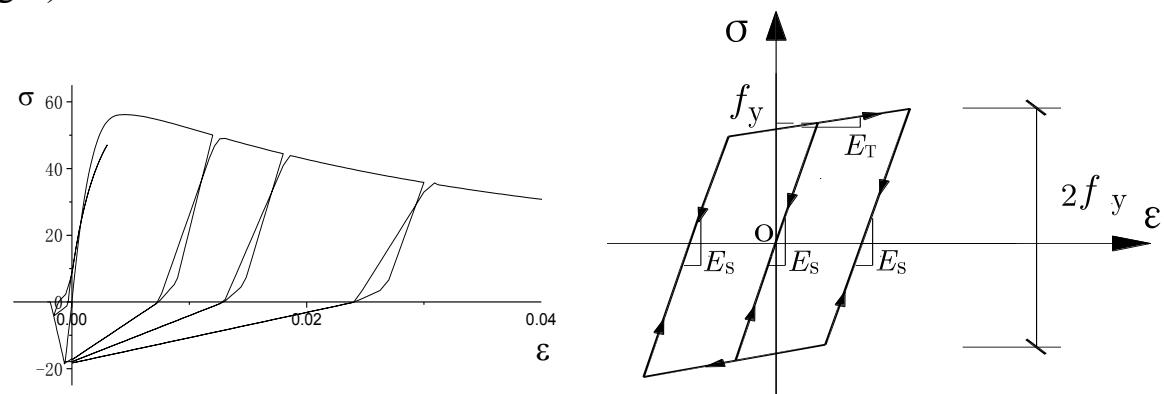

Fig.9 The Uniaxial Model of Concrete

Fig.10 The Uniaxial Model of Reinforcement

\section{The Uniaxial Constitutive Model of Reinforcement}

In reinforced concrete structures, the reinforcement are mainly bearing tension loading, and important to nonlinear characteristic of structure, the constitutive characteristic of reinforcement is simpler relative to concrete. In order to accurately describe the reinforcement characteristic, Bauschinger's Effect should be described exactly, that is the yield strength of reinforcement is decreased if reloading after yield. Typical reinforcement constitutive models are Menegotto-Pinto model(1973)[9], Kato model(1979) [10] and Dodd model(1995)[11], and so on. In order to simplify analysis, many scholars change the curves of reinforcement constitutive models to straight lines, and proposed some simple models, among which the linear hardening model, as shown in Fig.10, is widest applied for high efficiency and well describing the characteristic of reinforcement under hysteretic loading. 


\section{Conclusion}

The studies on uniaxial constitutive models of reinforcement and concrete are summarized, the typical concrete constitutive models are compared in terms of mechanic characteristic of reinforce confine concrete and hysteretic rule, and a practical model are proposed.

It is considered that the reinforcement constitutive model should describe Bauschinger's Effect, and a simple and practical bilinear strain hardening model is suggested.

The dynamic trend of the constitutive models development is introduced, and it is recognized that through the viscous of damage variables to consider strain rate effect is more reasonable.

\section{References}

[1]Guo Zhenhai, Shi Xudong. Reinforced concrete theory and analyse. beijin: Tsinghua University Press, 2003.

[2]Zhu Bolong, Dong Zhenxiang, Nonlinear analysis of reinforced concrete. Tongji University Press, 1985.11.

[3]GUO Zhenhai, ZHANG Xiuqin. Experimental study on complete stress2strain curves of concrete [J]. J Building Structures, 1982, 3 (1) : 112. ( in Chinese).

[4]R.W.G.B1akeley, R.Park, Prestressed concrete sections with cyclic flexures. Journal of Structural Engineering. ASCE,1973.

[5]J.B.Mander, M.J.N. Priestly, and R.Park, Theoretical Stress-Strain Model for Confined Concrete Journal of Structural Division, ASCE,Vol.114, No.8, pp.1804-1826,August, 1988.

[6]Teng zhiming, Zou lixiang.Nonlinear finite element analysis of rc members under reversed cyclic loading. China Civil Engineering Journal.1996 29(2):19-27.

[7]M. Saatcioglu, R.Razvi, Strength and ductility of confined concrete. ASCE J Structure Engineering , 1992, 118(6) : 1590-1607.

[8] Hu Qi, Yungui Li, Xilin Lv. A PRACTICAL CONFINED CONCRETE CONSTITUTIVE MODEL UNDER UNIAXIAL HYSTERESIS LOAD. ENGINEERING MECHANICS, 2011, 28(9): 95-102.

[9]M.Menegotto, P.Pinto, Method of Analysis for Cyclically loaded RC plane Frame including changes in geometry and non-elastic behavior of elements under combined normal force and bending. Symposium of resistance and ultimate deformatbility of structures acted on by well defined repeat loads IABSE report Vol 13 Lisbon 1973.

[10]B.Kato, Mechanical prorerties of steel under load cycles idealizing seismic action. AICAP-CEB sympisium.Rome. 1979.

[11]L.L.Dodd, J.I.Restrepo-Posada. Model for predicting cyclic behavior of reinforcing steel, Journal of structural engineering , 1995, 121(3): 433-445. 